\title{
Late presenting bilateral squamosal synostosis
}

Jason Diab,

Peter J. Anderson, Mark H. Moore

Australian Craniofacial Unit, Adelaide, Australia
Premature fusion of one or other of the minor sutures can subtly influence the shape of the human skull. Although infrequently reported or not clinically recognized, it can such contribute to a variety of craniofacial dysmorphisms. We herein report a case of late presenting, isolated bilateral synostosis of the squamosal suture dysmorphologies whose presentation mimics aspects of sagittal synostosis.

Keywords: Cranial sutures / Craniofacial abnormalities / Craniosynostosis

\section{INTRODUCTION}

The presentation of major cranial suture fusion is well recognized, but isolated minor craniosynostosis can present in diverse and unrecognized ways. This has been attributed to the increasing number of case reports presented by the community about the minor sutures. This case reports a late presenting isolated bilateral synostosis of the squamosal suture whose presentation was different and mimicked aspects of sagittal synostosis.

\section{CASE REPORT}

A 6-year-old boy presented to the unit from the pediatric outpatient for evaluation of his abnormal head shape. The fifth child in the family with no family history of craniofacial anomalies, he has a past medical history of autism, developmental delay and speech delay. The antenatal history was a full-term pregnancy with no perinatal or post-natal complications. Clinical examination identified a mild frontal prominence with a palpable bitemporal saddle like depression and some superior occipital fullness (Fig. 1). These findings were confirmed on a three-dimensional reconstructive computed tomography, which also showed symmetric bilateral squamosal synostosis

Correspondence: Jason Diab

The Australian Craniofacial Unit, 72 King William Rd, Adelaide 5006, Australia E-mail: jdmisciali@gmail.com

Received February 10, 2019 / Revised March 19, 2019 / Accepted March 21, 2019
(Fig. 2). All other major cranial sutures and fontanelles were unremarkable with a patent sagittal suture. There was no occipital bullet or flattening (Fig. 3).

He completed a multidisciplinary team assessment, followed by examination and investigations confirming no evidence of raised intracranial pressure. A bitemporal saddle like deformity was identified as a consequence of the premature fusion of the squamosal sutures. There were no significant changes in the cranial bases. He has since been managed conservatively with regular follow up without evidence of worsening head shape or neurodevelopmental delay.

\section{DISCUSSION}

Synostosis of the major cranial sutures can be readily diagnosed from the combination of clinical findings and computerized tomography scan images. By contrast in isolated minor suture craniosynostosis, such as the squamotemporal suture, the clinical manifestations are not so well recognized, and a more comprehensive analysis of imaging is often required. The squamosal suture lies between the squamous portion of the temporal bone and parietal bones extending posteriorly from the pterion into the parietomastoid suture [1]. The squamosal suture has both a temporal component with neural crest derived mesenchymal cells and a parietal component with mesoderm derived structures respectively [2]. Unlike other sutures, the squamosal closes in adult life at about the fourth decade. With improved imag- 

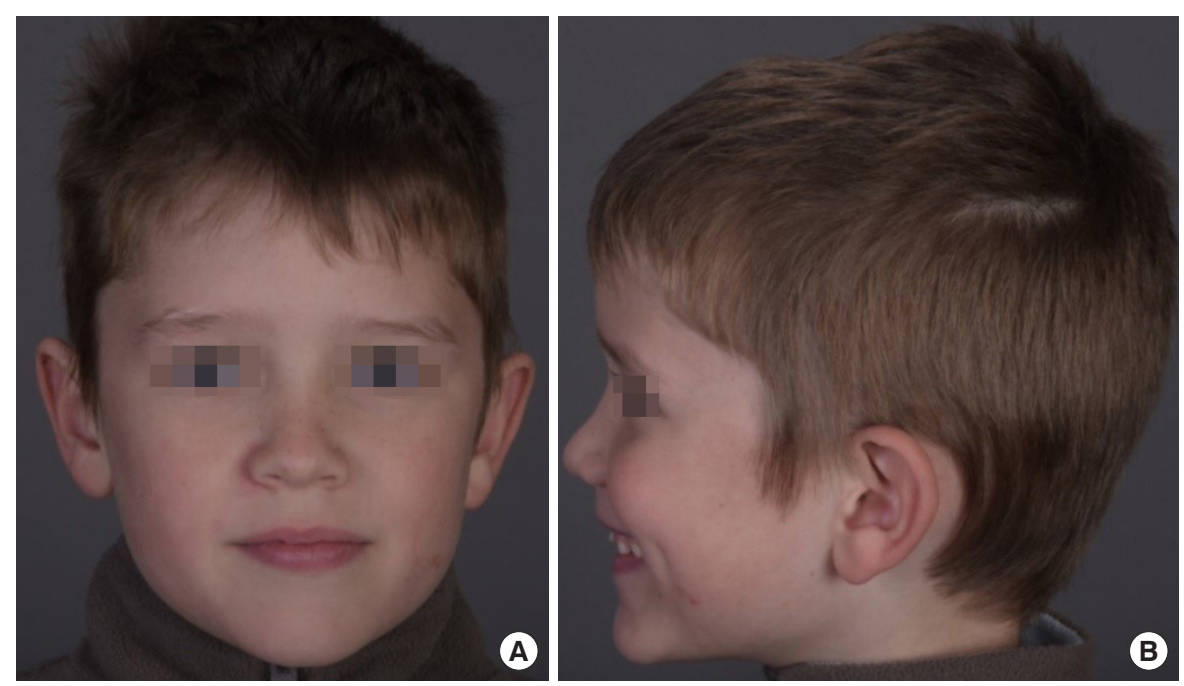

Fig. 1. A 6-year-old boy with bilateral squamosal synostosis. (A) Frontal view. (B) Lateral view.
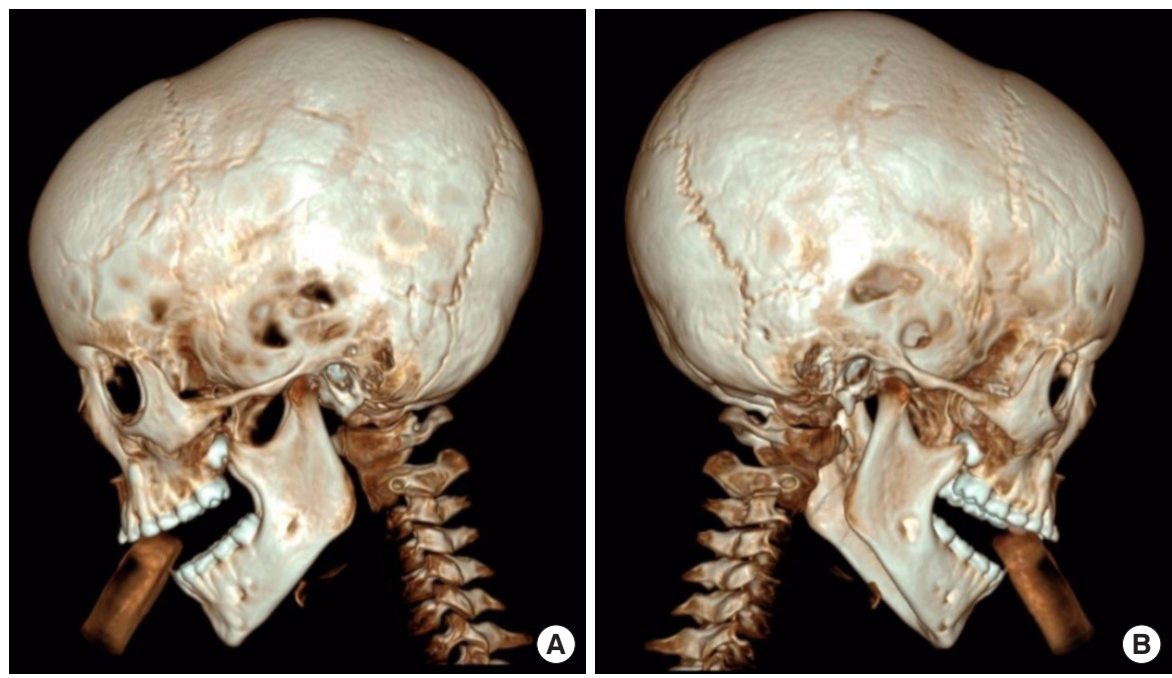

Fig. 2. Computerized tomography scan with three-dimensional reconstruction showing synostosis of left (A) and right (B) squamosal sutures.

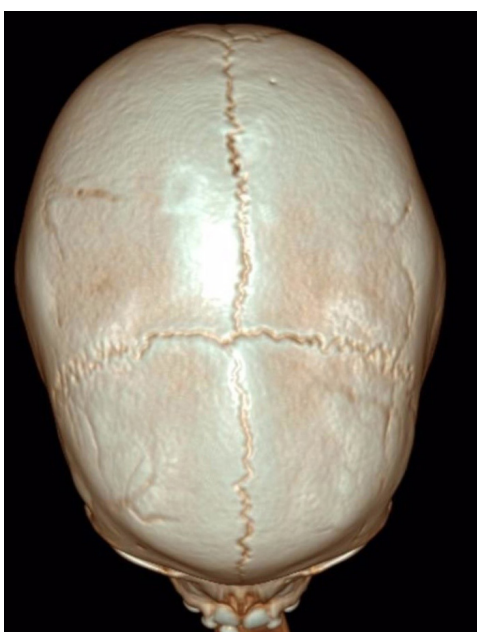

Fig. 3. Computerized tomography scan with three-dimensional reconstruction showing no occipital bossing or frontal bossing. ing and attention from the craniofacial surgical community, the literature would suggest an increased finding for this rare occurrence with great variation in phenotypic presentations in syndromic and non-syndromic patients [3]. Eley et al. [4] suggest that one may be under estimating or not detecting these minor suture involvements and their role in craniofacial development. Smartt et al. [5] reported the largest cohort study of squamosal synostosis, in which they highlight the varied nature of presentation with many presenting as part of a multisuture synostosis pattern. His isolated bilateral synostosis cases both presented with some form of occipital flattening.

Our case showed clinical findings and radiological findings consistent with Virchow's Law-growth restriction perpendicular to suture with compensation in parallel to the suture, albeit minor in its expression. It did not manifest the classical appear- 
ance as previously described. This case presented at 6 years of age compared to the much younger age of less than 2 years reported by Smartt. This case confirms phenotypic variation in non-syndromic isolated squamosal synostosis, one should consider this as a possible cause for atypical findings presenting with subtle changes in cranial morphology. Squamosal synostosis is not well recognized and can mimic a late presenting sagittal synostosis, but its influence on the skull morphology should raise the suspicion to clinicians. A comprehensive clinical assessment and radiological interpretation should be considered in the assessment protocol to exclude any minor suture involvement that may contribute to the cranial dysmorphism.

In this case, surgical intervention was not indicated in the absence of significant craniofacial dysmorphology or evidence of raised intracranial hypertension contributing to neurodevelopmental delay. There may be indications for surgical correction of more complex multisuture synostosis cases where correction can be considered in relation to major and minor sutures collectively.

\section{NOTES}

\section{Conflict of interest}

No potential conflict of interest relevant to this article was reported.

\section{Ethical approval}

The study was approved by the Institutional Review Board of the South Australian Women and Children's Health Network
Human Research Ethics Committee (approval No. 969A). Written informed consent was obtained.

\section{Patient consent}

The patient's parent provided written informed consent for the publication and the use of his images.

\section{ORCID}

Jason Diab https://orcid.org/0000-0001-7043-4224

Peter J. Anderson https://orcid.org/0000-0001-7430-868X

Mark H. Moore https://orcid.org/0000-0003-2136-0315

\section{REFERENCES}

1. Standring S. Gray's anatomy: the anatomical basis of clinical practice. 40th ed. New York: Churchill Livingstone; 2008.

2. Sperber GH. Craniofacial development. Hamilton: Decker; 2001.

3. Ranger A, Chaudhary N, Matic D. Craniosynostosis involving the squamous temporal sutures: a rare and possibly underreported etiology for cranial vault asymmetry. J Craniofac Surg 2010;21:1547-50.

4. Eley KA, Thomas GP, Sheerin F, Cilliers D, Wall S, Johnson D. The significance of squamosal suture synostosis. J Craniofac Surg 2016;27:1543-9.

5. Smartt JM Jr, Singh DJ, Reid RR, Hellinger JC, Hsu VM, Bartlett SP. Squamosal suture synostosis: a cause of atypical skull asymmetry. Plast Reconstr Surg 2012;130:165-76. 